\title{
In this issue: the editor's two cents
}

It is customary for reviewers to offer comments for the editor to help decide the fate of manuscripts. All papers that we ultimately publish undergo this tough scrutiny. But the last word always belongs to the editor. With this issue I am restoring a column that will consist of a few brief comments about each of the papers. Depending on the reaction from readers, this will either be a regular feature or only emerge when the spirit moves me and space permits.

The never-ending debate continues: in the OpinionDissent columns, supporters and critics of bike helmets square off again ( $p$ 86). This time, however, the focus is on an issue which, though exemplified well by the helmet controversy, is much broader: risk compensation. This too is being revisited, and, as before, both sides score well. Clearly, this is an area desperate for more research, or at least a systematic review.

Several papers address violence prevention. That by Reza, Mercy, and Krug (p 104) is destined to be a classic. As the authors promise, it provides basic data on violence worldwide. These should serve as benchmarks against which future progress can be measured. It is reassuring that WHO is represented among the authors; its involvement is likely to be critical in ensuring that progress is made worldwide.

Nordstrom et al (p 112) call attention yet again to the astounding number of US households with guns and to the apparently cavalier manner in which many are stored. I was not surprised at the patterns reported by farm $v$ city, type of gun, or the association with alcohol abuse but was amazed that having taken a gun safety course was associated with a much higher prevalence of loaded, unlocked guns. Can this be another example of the paradox first noted with drivers' education?

The intriguing report by McPhillips, Gallaher, and Koepsell ( $p$ 150) points to an increase in risk of injuries among infants who are hospitalized in the first three months of age. It remains unclear what is responsible for this risk and, indeed, how best to use the information for prevention. If anticipatory guidance was truly effective, this group could easily be targeted. But I remain sceptical...

Jain and colleagues (p 135) also focus on infant deaths. Although it has been shown previously that both poverty and low birth weight increase risk of injuries among infants, and are related to one another, the separate effects of race have not previously been documented. This study shows that there are different consequences for each of the main racial groups in the US.

Gerberich et al ( $\mathrm{p}$ 117) present results from a large sample of under-20s in farm households that show how frequently this population is injured. Although most of us appreciate how dangerous farm work can be, especially for young workers, the data serve to remind that many more are injured by non-farming events and that as is true for most other populations, sports and recreation are especially dangerous.

What might be viewed as a companion piece to the above is the paper by Pickett et al ( $\mathrm{p}$ 123) presenting findings from a national agricultural surveillance program in
Canada. It complements Gerberich's findings by pointing to the vulnerability of those over age 60 . Among farm related injuries in both studies, equipment and falls dominate. The former can be controlled through stricter regulation; but what to do about the latter?

Some lessons have to be learned repeatedly and some reside in folk wisdom. Chen, Braver, Baker, and $\mathrm{Li}$ (p 129) offer convincing evidence that young teens are dangerous drivers (old message), and even more dangerous when accompanied by teenage passengers (folk knowledge). Not surprising, but necessary to document in this elegant manner as a basis for the sort of restrictions transport safety officials should carefully consider.

Laflamme and Menckel's study (p 146) shows that school principals' assessment of the physical and psychosocial environment of their schools correlate well with the risk of injury. Although this may not be surprising, the possibly overlooked reminder that principals are a useful source of safety information seems compelling. They also may have much to offer to prevent injuries from physical violence (though the results do not directly support this belief).

Lightstone et al (p 155) describe how using geographic information system software can help understand patterns in child pedestrian injuries. This is perhaps best viewed as a work in process: the actual preventive implications differ little from studies using other methods, but mapping is part of the wave of the future and is likely to help understand why some locales are more dangerous than others.

An equally important bit of newish technology is that devised by Ellen Mackenzie's team to convert ICD injury codes to AIS scores. What Durbin, Localio, and MacKenzie have now done is to provide information about the validity of this process for children ( $p$ 96). This is an elegant paper with a reassuring message for all who use the ICD/AIS MAP software.

Another methodological contribution is that of Kendrick and Marsh ( $p$ 100) who answer a question that often troubles survey researchers: are responders and nonresponders likely to offer similar responses to safety practice questions? The answer in general is "yes, they are" but, hardly surprising, where differences were found responders were more likely to report taking recommended safety measures. Survey researchers need to remain on their toes.

More on helmets: I resolved not to take a position on the erudite discussion of the risk compensation question in this issue's opinion/dissent columns (see my comments above). But I could not resist bringing the following to our readers' attention: the May issue (Vol 33, No 3) of Accident Analysis and Prevention includes a meta-analysis of bicycle helmet efficacy (Attewell, Glase, and McFadden). Their conclusion? "The evidence is clear that bicycle helmets prevent serious injury and even death .... the summary odds ratio estimate for efficacy is 0.40 for head injury, 0.42 for brain injury, 0.53 for facial injury and 0.27 for fatal injury". As all confidence intervals exclude 1 , this figure indicates statistically significant protective effects. 